\title{
Acupuncture for lateral epicondylitis (tennis elbow): study protocol for a randomized, practitioner-assessor blinded, controlled pilot clinical trial
}

Kyung-Min Shin ${ }^{1}$, Joo-Hee Kim¹, Seunghoon Lee ${ }^{1}$, Mi-Suk Shin¹, Tae-Hun Kim², Hyo-Ju Park', Min-Hee Lee', Kwon-Eui Hong ${ }^{3}$, Seungdeok Lee ${ }^{4}$ and Sun-Mi Choi ${ }^{*}$

\begin{abstract}
Background: Lateral epicondylitis is the most frequent cause of pain around the elbow joint. It causes pain in the region of the elbow joint and results in dysfunction of the elbow and deterioration of the quality of life. The purpose of this study is to compare the effects of ipsilateral acupuncture, contralateral acupuncture and sham acupuncture on lateral epicondylitis.

Methods/design: Forty-five subjects with lateral epicondylitis will be randomized into three groups: the ipsilateral acupuncture group, contralateral acupuncture group and the sham acupuncture group. The inclusion criteria will be as follows: (1) age between 19 and 65 years with pain due to one-sided lateral epicondylitis that persisted for at least four weeks, (2) with tenderness on pressure limited to regions around the elbow joint, (3) complaining of pain during resistive extension of the middle finger or the wrist, (4) with average pain of NRS 4 or higher during the last one week at a screening visit and (5) voluntarily agree to this study and sign a written consent. Acupuncture treatment will be given 10 times in total for 4 weeks to all groups. Follow up observations will be conducted after the completion of the treatment, 8 weeks and 12 weeks after the random assignment. Ipsilateral acupuncture group and contralateral acupuncture group will receive acupuncture on LI4, TE5, LI10, LI11, LU5, LI12 and two Ashi points. The sham acupuncture group will receive treatment on acupuncture points not related to the lateral epicondylitis using a non-invasive method. The needles will be maintained for 20 minutes. The primary outcome will be differences in the visual analogue scale (VAS) for elbow pain between the groups. The secondary outcome will be differences in patient-rated tennis elbow evaluation (PRTEE), pain-free/maximum grip strength (Dynamometer), pressure pain threshold, clinically relevant improvement, patient global assessment, and the EQ-5D. The data will be analyzed with the paired $t$-test and ANCOVA $(P<0.05)$.
\end{abstract}

Discussion: The results of this study will allow evaluation of contralateral acupuncture from two aspects. First, if the contralateral acupuncture shows the effects similar to ipsilateral acupuncture, this will establish clinical basis for contralateral acupuncture. Second, if the effects of contralateral acupuncture are not comparable to the effects of ipsilateral acupuncture, but are shown to be similar to the effects of the sham acupuncture, we can establish the basis for using the same acupoints of the unaffected side as a control in acupuncture clinical studies.

Trial registration: This trial has been registered with the 'Clinical Research Information Service (CRIS)', Republic of Korea: KCT0000628.

Keywords: Acupuncture, Tennis elbow, Epicondylitis

\footnotetext{
* Correspondence: smchoi@kiom.re.kr

'Department of Medical Research, Korea Institute of Oriental Medicine, Daejeon, South Korea

Full list of author information is available at the end of the article
} 


\section{Background}

Lateral epicondylitis is a disease that can result in elbow joint pain and dysfunction, and decrease in the quality of life [1]. Lateral epicondylitis is the most common cause of pain around the elbow joint, and occurs because of overuse injury and repetitive stress [1,2]. Every year, about 1 to $3 \%$ of the general population is affected [3], and there is no difference in morbidity between the sexes, but the prevalence rate is higher in people over 40 years of age [4]. In addition, it is more frequent in the dominant arm [5], and if untreated the symptoms persist for 6 to 24 months [6-8].

Treatment for lateral epicondylitis includes non-steroidal anti-inflammatory drugs (NSAIDs), corticosteroid injection, botulinum toxin injection, extracorporeal shock wave therapy, low-level laser therapy, physiotherapy, acupuncture, and surgery [4]. The short-term effect of ultrasound therapy [9], low-level laser therapy [10], and acupuncture $[11,12]$ has been accepted, but there is insufficient evidence regarding their long-term effects [13]. Further, the shortterm effects of injection treatments have been accepted, but problems such as adverse events and high frequency of relapse, still exist [14-16].

Previous studies of lateral epicondylitis include a case study of fire needle therapy [17], and randomized controlled trials (RCTs) comparing the effects of acupuncture against sham acupuncture [18-20], physical therapy [21], and electro-acupuncture [22]. Sham acupuncture, which is used in clinical studies of acupuncture, includes invasive and noninvasive methods. The acupoint used as the control includes the actual acupoint used in the treatment, areas away from the actual acupoint (non-acupoint), or acupoints unrelated to the specific indication. Sham acupuncture used as controls in previous studies of lateral epicondylitis includes using areas away from the acupoint (non-acupoint) [18], minimal acupuncture at the same acupoint [20], and non-invasive acupuncture at an unrelated acupoint [19]. However, in clinical studies of acupuncture, one of the problems of using sham acupuncture is that practitioner blinding is not done.

Contralateral acupuncture is a traditional acupuncture technique, whereby acupuncture points on the right side are selected for diseases or disorder on the left and vice versa [23]. Several studies have shown effects of contralateral acupuncture on hemihidrosis [24], herpes zoster [24], dizziness [25], pain [24,26-29] and stroke [30]. Furthermore, there have been studies comparing the brain function [31], the change ratio of mean blood flux [32], and the blood volume [33] of the needle-inserted side and the side without insertion after insertion at acupoints, such as the LI4 and ST36, on normal subjects. However, there have not been any studies on lateral epicondylitis comparing ipsilateral acupuncture and contralateral acupuncture at the same acupoints.
Therefore, this study is a randomised, practitionerblinded, parallel-group, sham-controlled pilot study of patients with lateral epicondylitis, to compare the efficacy of ipsilateral acupuncture, contralateral acupuncture, and sham acupuncture (non-invasive acupuncture at an unrelated acupoint). Especially, this study is designed to conduct a practitioner-blinded study by setting up separate groups of diagnosticians and practitioners in an attempt to examine the possibility of a practitioner-blinded clinical trial.

\section{Methods/design}

Aim of the study

The aim of this pilot study is to assess the efficacy of unaffected-side acupuncture by comparing the efficacy of ipsilateral acupuncture, contralateral acupuncture and placebo acupuncture in patients with lateral epicondylitis. And through this study, we will evaluate the possibility of a practitioner-blinded clinical trial.

\section{Study design}

This study is a randomised, practitioner-blinded (to affected side), parallel-group, placebo-controlled pilot study of patients with lateral epicondylitis. The subjects who voluntarily sign a consent form will undergo the examinations according to the clinical study design. When a subject is determined to be appropriate for participation based on the inclusion and exclusion criteria, the subject will be randomly assigned to one of three groups during the second visit (Table 1). Treatments will be performed two to three times a week. All three groups receive 10 acupuncture treatments over 4 weeks. A once-a-week treatment and a four-times-aweek treatment will be permitted to minimize the differences in the total number of treatments. Follow up observations will be conducted at the end of 4, 8 and at 12 weeks after randomized assignment. The affectedand unaffected-side acupoints were selected based on acupuncture literature and prior studies $[18,20,21]$. The acupoints for the control group were selected after referring to a previous study [19], with six acupoints added to maintain the same number of treated acupoints between the groups. To summarize, all three groups will receive acupunctures at eight acupoints (Figure 1).

The practitioner and diagnostician have had more than 4 years of clinical experience since completing a 6-year-long college of Korean medicine and have received a Doctor of Korean Medicine.

\section{Randomization and allocation concealment}

This study is designed as a pilot study and allowing for a $20 \%$ dropout rate, each group will include 15 participants [34,35]. Participants will be assigned with a 1:1:1 allocation ratio according to a computer-generated 
Table 1 Patient inclusion and exclusion criteria

Inclusion criteria

1. Individuals between the ages of 19 and 65 years with lateral epicondylitis on one arm and pain persisting for at least 4 weeks

2. Individuals with tenderness limited to the elbow joint and surrounding area

3. Individuals reporting pain under resisted extension of the middle finger and wrist

4. Individuals with an average pain of 40 or more (0 to 100) on the visual analogue scale) in the week prior to the screening visit

5. Individuals who volunteered to participate in the study and who signed a consent form

\section{Exclusion criteria}

1. Individuals whose radiological examinations show abnormalities such as calcification, arthritis, and inflammatory arthropathy of the elbow joint

2. Individuals with a history of trauma, ligament damage, fracture, tumor, or surgery of the elbow joint

3. Individuals who have been diagnosed with or treated for cervical radiculopathy or herniation of intervertebral disc

4. Individuals who have received injections for lateral epicondylitis during the last 6 months

5. Individuals who have received treatments such as non-steroidal antiinflammatory drugs, acupuncture, physiotherapy for lateral epicondylitis during the last 2 weeks

6. Individuals judged by the person in charge of the clinical trial as unsuitable for participation, such as those with mental disorders, who are pregnant, or have other acute or chronic disorders randomization list. Randomization will be stratified by dominant hand, as left- or right-handed. The allocation will be concealed in sequentially numbered, opaque, sealed envelopes and the random allocation envelops will be opened only after the participant has satisfied all selection criteria and completed baseline assessments. The participants and diagnostician will know the allocated group but the practitioners, outcome assessors and data analysts will not.

\section{Practitioner blinding}

Practitioner blinding will be confirmed by separately involving diagnosticians and practitioners in the study. The practitioner will not participate in the screening and random assignment so will be unaware of the affected side. On screening and before each acupuncture treatment session, the diagnostician will assess the Ashipoints of the subjects. The diagnostician will mark and describe the subjects' Ashi-points on a diagram of the

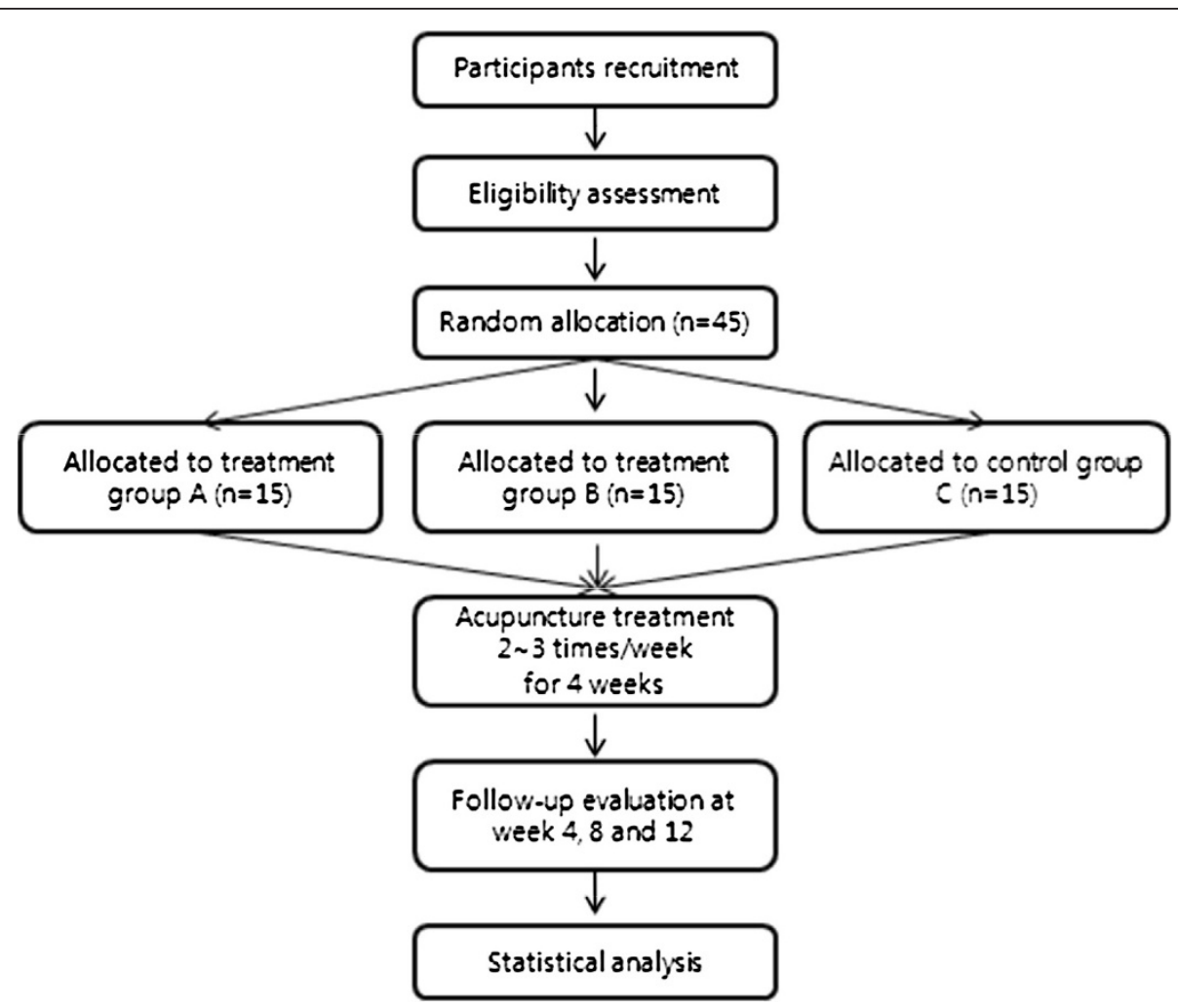

Figure 1 Flowchart of the study design. 
arm, and then the practitioner will perform acupuncture after confirming only the side assigned for treatment. Appropriateness of practitioner blinding will be assessed after the end of the treatment.

\section{Ethics}

This protocol adheres to the principles of the Declaration of Helsinki and has been approved by the institutional review boards of the Daejeon University Hospital, where the study will take place (djomc-100-1), and written consent will be obtained from each participant before any treatment is given. All patients will have the right to withdraw from the study at any time.

\section{Inclusion criteria}

1. Individuals between the ages of 19 and 65 years with lateral epicondylitis on one arm and pain persisting for at least 4 weeks.

2. Individuals with tenderness limited to the elbow joint and surrounding area.

3. Individuals reporting pain under resisted extension of the middle finger and wrist.

4. Individuals with an average pain of 40 or more $(0-100)$ on the visual analogue Scale (VAS) in the week prior to the screening visit.

5. Individuals who volunteered to participate in the study and who signed a consent form.

\section{Exclusion criteria}

1. Individuals whose radiological examinations show abnormalities such as calcification, arthritis, and inflammatory arthropathy of the elbow joint.

2. Individuals with a history of trauma, ligament damage, fracture, tumor, or surgery of the elbow joint.

3. Individuals who have been diagnosed with or treated for cervical radiculopathy or herniation of intervertebral disc.

4. Individuals who have received injections for lateral epicondylitis during the last 6 months.

5. Individuals who have received treatments such as non-steroidal anti-inflammatory drugs (NSAIDs), acupuncture, physiotherapy for lateral epicondylitis during the last 2 weeks.

6. Individuals judged by the person in charge of the clinical trial as unsuitable for participation, such as those with mental disorders, who are pregnant, or have other acute or chronic disorders.

\section{Interventions}

\section{Ipsilateral acupuncture group}

The subjects will receive 10 treatments for 4 weeks. Treatments will be performed two to three times a week, at a minimum of one per week and a maximum of four per week. The acupoints that will be used are as follows: LI4, TE5, LI10, LI11, LU5, LI12, and two Ashi-points. Sterilized disposable acupuncture needles of size $0.25 \times$ $30 \mathrm{~mm}$ will be used. Deqi will be induced by manual stimulation, where the needles will be inserted for 20 minutes and then removed.

\section{Contralateral acupuncture group}

The subjects in the contralateral acupuncture group will receive the same frequency, duration, and total number of treatments as the ipsilateral acupuncture group. Needles will be inserted at the same acupoints of the unaffected side by using the same manual technique and retaining time.

\section{Control group}

For the control group, the acupoints used (BL13, Bl14, $\mathrm{Bl} 15$, and Bl16 of both the sides) are unrelated to the treatment of lateral epicondylitis, and non-invasive techniques will be used, namely, the Park sham placebo acupuncture device [36]. The subjects in the control group will receive the same frequency, duration, and total number of treatments and retaining time as the ipsilateral and contralateral acupuncture groups.

\section{Prohibited concomitant treatments}

During the treatment period, the subjects will be prohibited from receiving any concomitant treatments for the lateral epicondylitis. And the subjects will be allowed to decide whether to receive additional treatment during the assessment period. Any other related treatments will be recorded in detail.

\section{Outcomes}

\section{Primary outcome measure}

The primary outcome measure in this study is the difference in visual analog scale (VAS) for elbow pain between the groups at 4 weeks after random assignment. The intensity of perceived pain of the elbow in the past week will be measured using a VAS consisting of a 100-mm horizontal line, where the left end indicates no pain, and the right end indicates maximum pain.

\section{Secondary outcome measures}

The secondary outcome measures are the difference in VAS for elbow pain between the groups at 8 and 12 weeks after random assignment, and the differences in patient-rated tennis elbow evaluation (PRTEE), painfree/maximum grip strength, pressure pain threshold, clinically relevant improvement, patient global assessment and EuroQol between the groups at 4, 8, and 12 weeks after random assignment.

PRTEE, a tool specifically developed to assess lateral epicondylitis [37], will be used. PRTEE is composed of 
two parts: assessment of pain and function [38]. Painfree/maximum grip strength will be measured using a dynamometer (Baseline ${ }^{\bullet}$ Digital Smedley Spring Dynamometer, Fabrication Enterprises, Inc., Irvington, New York, USA). The subjects will be asked to take a shoulder-width stance and allow their arms to hang loose. The pain-free grip strength will be measured, followed by the measurement of the maximum grip strength, and the affected side will be measured first and then the unaffected side. The measurement readings will be not revealed to the subjects until the completion of the test. The pain-free grip strength will be measured up to the point when the subject feels uneasy. The maximum grip strength will be measured at the maximum grip level. While administering a perpendicular pressure on the common extensor tendon area with a maximum pressure of $6 \mathrm{~kg} / \mathrm{cm}^{2}$ by using a pressure algometer (PainTest ${ }^{\mathrm{Tw}}$ FPX 25 Algometer, Wagner Instruments, Greenwich, CT, USA), the measurement will be taken at the moment when the sensation of pressure changes to pain. The mean value of three measurements taken at an interval of 20 seconds will be used [39]. Clinically relevant improvement will be defined when a 50\% decrease in VAS is observed before and after the treatment. Quality of life will be measured using the Korean version of the EuroQol-5D (EQ-5D) tool [40]. Self-perceived improvements will be assessed by comparing sensations before and after the treatment using the patient global assessment (PGA) scale [41]. Subjects can select one of the following five responses to describe the improvement of symptoms when compared to that before the treatment: greatly improved; somewhat improved; no changes; somewhat exacerbated; and greatly exacerbated. A varied and translated version of the psychometric properties of the credibility/expectancy questionnaire [42] will be used to measure the credibility and expectancy of the subjects. From this questionnaire, the expectations of the subjects in relation to acupuncture therapy can be determined. Furthermore, differences in acupuncture efficacy depending on expectations can be assessed. In this study, we will be using the Southampton Needle Sensation Questionnaire [43] to assess the sensation that the subjects experience after the first acupuncture treatment. To determine whether practitioner blinding will be done appropriately, the practitioners will be asked to guess the group to which each of the subjects belongs.

\section{Follow up}

Follow up observations will be conducted at 8 and 12 weeks after the random assignment.

\section{Statistical analysis}

Analysis of covariance (ANCOVA) will be performed, with the primary analysis variables and relative changes in the values of secondary analysis variables, at the 4th, 8th and 12th week, compared to the baseline values, as dependent variables. The baseline value will be set as a covariate and the group as the fixed factor. The statistical significance threshold will be set at 0.05 (two-sided), with the $95 \%$ confidence interval. Missing data will be handled with the mixed model for repeated measures (MMRM) under the assumption that observations are missing at random (MAR). Safety analysis will be performed by analyzing the frequency of adverse events suspected as related to the treatment, and serious adverse events. The data for adverse events will be collected through the symptoms reported by the patients, and observations by a researcher at every visit. Among the variables for the primary outcome, sub analysis can be performed to determine whether the pain area of the subjects, faith in acupuncture treatment, and credibility of the efficacy of acupuncture treatment affect actual improvements in pain. Regression analysis can be performed to find the variables that have an influence on pain reduction. We will use the $\mathrm{SAS}^{\circ}$ version 9.3 (SAS institute. Inc., Cary, NC, USA) for statistical analysis.

\section{Adverse events}

The subjects will be requested to voluntarily report information about adverse events, and the researcher will confirm the occurrence of adverse events through methods such as a medical interview. Details about adverse events, such as the date of occurrence, lost time, degree of adverse events, measures taken related to the treatment, causal relationship with the treatment, other treatments or medications that are suspected to cause the adverse event, and treatment of the adverse event, will be recorded in detail.

\section{Discussion}

By using the results of our study, we plan to evaluate two aspects of contralateral acupuncture. First, if the contralateral acupuncture shows similar efficacy to the ipsilateral acupuncture, we can establish the clinical basis for contralateral acupuncture. Second, if contralateral acupuncture is not as efficacious as ipsilateral acupuncture and is similar to sham acupuncture, then we can establish the basis for using the same acupoints of the unaffected side as a control in acupuncture clinical studies. In other words, in diseases where either one of the left or right sides is affected, such as diseases of the shoulder joint, elbow joint, knee joint, or ankle joint, the contralateral acupuncture could serve as areas where sham acupuncture can be performed and can allow double-blinded studies.

\section{Trial status}

This trial is currently recruiting participants.

\section{Abbreviations}

ANCOVA: Analysis of covariance; EQ-5D: EuroQol-5D; MAR: Missing at random; MMRM: Mixed model for repeated measures; NSAID: Non-steroidal anti- 
inflammatory drug; PGA: Patient global assessment; PRTEE: Patient-rated tennis elbow evaluation; RCT: Randomized controlled trial; VAS: Visual analog scale.

\section{Competing interests}

The authors declare that they have no competing interests.

\section{Authors' contributions}

KMS, SDL, and KEH participated in the design of the study, coordinate the study and drafted the manuscript. JHK, SHL, MSS, THK, HJP, MHL, and SMC provided technical advice and wrote the relevant sections of the manuscript. All authors participated in, read, and approved the final manuscript.

\section{Acknowledgements}

This study was supported by a grant of the Developement of Acupuncture, Moxibustion and Meridian Standard Health Technology, Korea Institute of Oriental Medicine, Republic of Korea (K12010).

\section{Author details}

${ }^{1}$ Department of Medical Research, Korea Institute of Oriental Medicine, Daejeon, South Korea. ${ }^{2}$ Clinical research center, MokHuri Neck\&Back Hospital, Seoul, South Korea. ${ }^{3}$ Department of Acupuncture \& Moxibustion Medicine, College of Korean Medicine, Daejeon University, Daejeon, South Korea. ${ }^{4}$ Department of Acupuncture \& Moxibustion Medicine, College of Korean Medicine, Dongguk University, Gyeongju, South Korea.

Received: 20 March 2013 Accepted: 31 May 2013

Published: 14 June 2013

\section{References}

1. Serafini G: Treatment of Lateral Epicondylitis. In Ultrasound-guided Musculoskeletal Procedures. Edited by Sconfienza LM, Serafini G, Silvestri E. Milan: Springer-Verlag Italia; 2012:67-71.

2. Krogh TP, Bartels EM, Ellingsen T, Stengaard-Pedersen $K$, Buchbinder R, Fredberg U, Bliddal H, Christensen R: Comparative effectiveness of injection therapies in lateral epicondylitis. Am J Sports Med 2012, 41:1435-1446.

3. Hamilton PG: The prevalence of humeral epicondylitis: a survey in general practice. J R Coll Gen Pract 1986, 36:464-465.

4. Johnson GW, Cadwallader K, Scheffel SB, Epperly TD: Treatment of lateral epicondylitis. Am Acad Fam Physicians 2007, 76:843-848.

5. Cohen M, Filho GRM: Lateral epicondylitis of the elbow. Revista Brasileira de Ortopedia 2012, 47:414-420.

6. Murtagh JE: Tennis elbow. Aust Fam Physician 1988, 17:90-91.

7. Hudak PL, Cole DC, Haines AT: Understanding prognosis to improve rehabilitation: the example of lateral elbow pain. Arch Phys Med Rehabil 1996, 77:586-593.

8. Cyriax JH: The pathology and treatment of tennis elbow. J Bone Joint Surg Br 1936, 18:921-940.

9. Smidt N, Assendelft W, Arola H, Malmivaara A, Green S, Buchbinder R, Windt D, Bouter $L$ : Effectiveness of physiotherapy for lateral epicondylitis: a systematic review. Ann Med 2003, 35:51-62.

10. Bjordal JM, Lopes-Martins RAB, Joensen J, Couppe C, Ljunggren AE, Stergioulas A, Johnson Ml: A systematic review with procedural assessments and meta-analysis of low level laser therapy in lateral elbow tendinopathy (tennis elbow). BMC Musculoskelet Disord 2008, 9:75.

11. Green S, Buchbinder R, Barnsley L, Hall S, White M, Smidt N, Assendelft WJJ: Acupuncture for lateral elbow pain. Cochrane Database Syst Rev 2002, 1, CD003527.

12. Trinh $\mathrm{KV}$, Phillips SD, Ho E, Damsma K: Acupuncture for the alleviation of lateral epicondyle pain: a systematic review. Rheumatology 2004, 43:1085-1090.

13. Chesterton LS, Mallen CD, Hay EM: Management of tennis elbow. Open Access J Sports Med 2011, 2:53-59.

14. Smidt N, Assendelft WJJ, van der Windt DAWM, Hay EM, Buchbinder R, Bouter LM: Corticosteroid injections for lateral epicondylitis: a systematic review. Pain 2002, 96:23-40.

15. Barr S, Cerisola FL, Blanchard V: Effectiveness of corticosteroid injections compared with physiotherapeutic interventions for lateral epicondylitis: a systematic review. Physiotherapy 2009, 95:251-265.
16. Viala CG, Dougados M, Gossec L: Efficacy and safety of steroid injections for shoulder and elbow tendonitis: a meta-analysis of randomised controlled trials. Ann Rheum Dis 2009, 68:1843-1849.

17. Lu C, Chiu HHE: Lateral epicondylitis successfully treated with fire needle therapy: A case report. Chin J Integr Med 2012, 18:395-397.

18. Fink M, Wolkenstein E, Luennemann M, Gutenbrunner C, Gehrke A, Karst M: Chronic epicondylitis: effects of real and sham acupuncture treatment: a randomised controlled patient-and examiner-blinded long-term trial. Forschende Komplementärmedizin/Research in Complementary Medicine 2002, 9:210-215.

19. Molsberger A, Hille E: The analgesic effect of acupuncture in chronic tennis elbow pain. Rheumatology 1994, 33:1162-1165.

20. Haker E, Lundeberg T: Acupuncture treatment in epicondylalgia: a comparative study of two acupuncture techniques. Clin J Pain 1990, 6:221-226.

21. Davidson $\mathrm{JH}$, Vandervoort A, Lessard L, Miller $\mathrm{L}$ : The effect of acupuncture versus ultrasound on pain level, grip strength and disability in individuals with lateral epicondylitis: a pilot study. Physiother Can 2001, 53:195-202.

22. Tsui $P$, Leung MC: Comparison of the effectiveness between manual acupuncture and electro-acupuncture on patients with tennis elbow. Acupunct Electrother Res 2002, 27:107-117.

23. Veith I: The yellow emperor's classic of internal medicine. Berkeley: University of California Press; 2002.

24. Lu F: Clinical application of contralateral acupuncture technique. J Tradit Chin Med 1997, 17:124-126.

25. Cheng B: 206 cases of spinogenic dizziness treated by contralateral acupuncture. J Tradit Chin Med 1996, 16:35-36.

26. Fang JQ, Liu YL, Mo XM: Clinical and experimental studies on analgesic effects of ipsilateral and contralateral stimulations with electro-acupuncture. Zhongguo Zhong Xi Yi Jie He Za Zhi 1994, 14:579-582.

27. Yang EJ, Koo ST, Kim YS, Lee JE, Hwang HS, Lee MS, Choi SM: Contralateral electroacupuncture pretreatment suppresses carrageenan-induced inflammatory pain via the opioid-mu receptor. Rheumatol Int 2011, 31:725-730.

28. Miura K, Ohara T, Zeredo JL, Okada Y, Toda K, Sumikawa K: Effects of traditional "Juci" (contralateral acupuncture) on orofacial nociceptive behavior in the rat. J Anesth 2007, 21:31-36.

29. Saidah R, Chueire AG, Rejaili WA, Peres NRB, SilvaV JBG, Schiavo F: Use of acupuncture after arthroscopic knee surgery and its relationship to pain, physical activity and need of walking aid. Acta Ortopédica Brasileira 2003, 11:5-10.

30. Kim M, Choi TY, Lee MS, Lee HS, Han CH: Contralateral acupuncture versus ipsilateral acupuncture in the rehabilitation of post-stroke hemiplegic patients: a systematic review. BMC Complement Altern Med 2010, 10:41.

31. Woo YM, Lee MS, Nam Y, Cho HJ, Shin BC: Effects of contralateral electroacupuncture on brain function: a double-blind, randomized, pilot clinical trial. J Altern Complement Med 2006, 12:813-815.

32. Guangjun W, Yuying T, Shuyong J, Tao H, Weibo Z: Change of blood perfusion in hegu acupoint after contralateral hegu acupoint was stimulated. J Altern Complement Med 2012, 18:784-788.

33. Kubo K, Yajima H, Takayama M, Ikebukuro T, Mizoguchi H, Takakura N: Changes in blood circulation of the contralateral achilles tendon during and after acupuncture and heating. Int J Sports Med 2011, 32:807-813.

34. Johanson GA, Brooks GP: Initial scale development: sample size for pilot studies. Educ Psychol Meas 2010, 70:394-400.

35. Julious SA: Sample size of 12 per group rule of thumb for a pilot study. Pharm Stat 2005, 4:287-291.

36. Park JB, White A, Stevinson C, Ernst E, James M: Validating a new non-penetrating sham acupuncture device: two randomised controlled trials. Acupunct Med 2002, 20:168-174.

37. Rompe JD, Overend TJ, MacDermid JC: Validation of the patient-rated tennis elbow evaluation questionnaire. J Hand Ther 2007, 20:3-10.

38. MacDermid JC: The Patient-Rated Tennis Elbow Evaluation (PRTEE) User Manual. Hamilton, Canada: School of Rehabilitation Science, McMaster University; 2007.

39. Smidt N, van der Windt DA, Assendelft WJ, Mourits AJ, Devillé WL, de Winter AF, Bouter LM: Interobserver reproducibility of the assessment of severity of complaints, grip strength, and pressure pain threshold in patients with lateral epicondylitis. Arch Phys Med Rehabil 2002, 83:1145-1150. 
40. Hurst NP, Kind P, Ruta D, Hunter M, Stubbings A: Measuring health-related quality of life in rheumatoid arthritis: validity, responsiveness and reliability of EuroQol (EQ-5D). Rheumatology 1997, 36:551-559.

41. Endicott J, Spitzer RL, Fleiss JL, Cohen J: The global assessment scale: a procedure for measuring overall severity of psychiatric disturbance. Arch Gen Psychiatry 1976, 33:766-771.

42. Devilly GJ, Borkovec TD: Psychometric properties of the credibility/ expectancy questionnaire. J Behav Ther Exp Psychiatry 2000, 31:73-86.

43. White P, Bishop F, Hardy H, Abdollahian S, White A, Park JB, Kaptchuk TJ, Lewith GT: Southampton needle sensation questionnaire: development and validation of a measure to gauge acupuncture needle sensation. J Altern Complement Med 2008, 14:373-379.

doi:10.1186/1745-6215-14-174

Cite this article as: Shin et al: Acupuncture for lateral epicondylitis

(tennis elbow): study protocol for a randomized, practitioner-assessor blinded, controlled pilot clinical trial. Trials 2013 14:174.

\section{Submit your next manuscript to BioMed Central and take full advantage of:}

- Convenient online submission

- Thorough peer review

- No space constraints or color figure charges

- Immediate publication on acceptance

- Inclusion in PubMed, CAS, Scopus and Google Scholar

- Research which is freely available for redistribution 